\title{
Insights on the application of some current SPH approaches for the study of muddy debris flow: numerical and experimental comparison
}

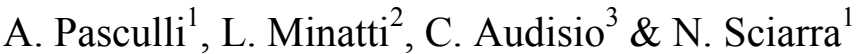 \\ ${ }^{1}$ University of Chieti-Pescara, Italy \\ ${ }^{2}$ University of Florence, Italy \\ ${ }^{3}$ Provincia di Torino, Italy
}

\begin{abstract}
The modeling of debris flow poses serious numerical problems, particularly with regard to the significant distortions of the numerical grid, as it is usually applied in both Eulerian and Lagrangian mesh based approaches. In alternative, meshless type techniques have been proposed by different authors. Among others, SPH (Smoothed Particle Hydrodynamics) is a purely mesh-free Lagrangian method well suitable for computing highly transitory free surface flows of complex fluids in complex geometries. However, a drawback of SPH method is the modelling of the interaction between the fluid and the solid boundaries, for which different approaches have been proposed. Accordingly, we developed a 2D SPH research numerical model, also in order to both implement different kind of SPH algorithms and perform sensitivity analysis based on experimental test outcomes, as already discussed previously. Hence the content of this paper is aimed at discussing further insights, in particular, about how much the numerical variability of the parameters requested to apply the selected Herschel-Bulkley non Newtonian constitutive rheological equations, describing a viscoplastic material like mudflows, affect the numerical results. Moreover further comparisons among numerical results are given and discussed.
\end{abstract}

Keywords: SPH, 2D numerical modeling, parametric studies, muddy debris flow.

\section{Introduction}

The SPH method is a numerical technique that was initially developed to solve astrophysical problems [1]. Its meshless and Lagrangian nature makes it very 
attractive for solving fluid flow problems where free surface boundary conditions and large strain rates are involved. Thus, this approach is particular attractive regarding the simulation, in particular, of fast muddy debris flow. On the other hand, some drawbacks should be properly addressed, in particular, in the application of this method to simulate the interaction of the flow with solid boundaries. So, in order to provide a flexible tool, suited for easy implementation and testing of different numerical SPH approaches proposed in literature, we developed a research code, written in 2005 Fortran language [2-4]. Then we performed many numerical parametric analyses compared with a selected laboratory experimental test [5], some of which were discussed in [4]. Some issues emerged, among which the influence of the numerical values uncertainty of the parameters of the rheological Herschel-Bulkley law on the calculated flow velocity. Therefore, in the following sections, after a brief summary of the selected mathematical and numerical approaches, insights coming from further numerical experiments are discussed.

\section{Mathematical modeling}

In order to study the flow of an incompressible non-Newtonian fluid we resort to the basic 'First Principles' governing the motion of a continuum, namely the mass and the momentum balance (Navier Stokes equations) in Lagrangian form as:

$$
\frac{D \rho}{D t}+\rho \nabla \cdot \underline{\mathbf{v}}=0 ; \rho \frac{D \underline{\mathbf{v}}}{D t}=\rho \underline{\mathbf{f}}+\nabla \cdot \underline{\boldsymbol{\sigma}}
$$

where $\underline{\mathbf{v}}$ is the local velocity of the continuum $\rho$ is the local density of the continuum; $\underline{\mathbf{f}}$ is the body force per unit of mass exerted on the continuum; $\underline{\boldsymbol{\sigma}}$ is the local total stress tensor. The stress tensor decomposition is indicated as follows in the paper: $\underset{=}{\boldsymbol{\sigma}}=-p \underline{\underline{\mathbf{I}}}+\underline{\underline{\boldsymbol{\tau}}}$, where: $p$ is the isotropic pressure; $\underline{\mathbf{I}}$ is the unit tensor; $\underset{\tau}{=}$ is the deviatory part of the total stress tensor. Regarding the constitutive law selected to simulate the behaviour of a mudflow, assumed as incompressible, we used the Herschel-Bulkley law, modified by Papanastasiou [6] in order to regularize the viscosity that, otherwise, would diverge to infinity for strain rate approaching to zero:

$$
\begin{aligned}
& \eta_{p}=K \sqrt{2 \operatorname{Tr}\left(\underline{\underline{\varepsilon}}^{2}\right)^{(n-1)}}+\frac{\tau_{c}}{\sqrt{2 \operatorname{Tr}\left(\underline{\underline{\varepsilon}}^{2}\right)}} \cdot\left\{1-\exp \left[-B \sqrt{2 \operatorname{Tr}\left(\underline{\underline{\varepsilon}}^{2}\right)}\right]\right\} \\
& \underline{\boldsymbol{\tau}}=2 \eta \underline{\underline{\varepsilon}} \text { if } \sqrt{\operatorname{Tr}\left({\underline{\underline{\boldsymbol{\tau}^{2}}}}^{2}\right) / 2}>\tau_{c} \quad \underline{\underline{\boldsymbol{\varepsilon}}}=\underline{\underline{\mathbf{0}}} \text { if } \sqrt{\operatorname{Tr}\left(\underline{\underline{\boldsymbol{\tau}}}^{2}\right) / 2} \leq \tau_{c}
\end{aligned}
$$

where $\eta_{p}$ is the local regularized dynamic viscosity of the fluid; $\tau_{c}$ is the yield stress; $K$ is called the liquid consistency; $n$ is called the power law index; $\underline{\boldsymbol{\varepsilon}}$ is the strain rate tensor. The physical meaning of the yield stress is immediate, representing the stress threshold below which the fluid starts to behave like a 
rigid body. The parameter $B$ (equal to 10 [s] for all simulations discussed in this paper) is related to the maximum viscosity returned by the regularization when the strain rate is zero. In this case the maximum viscosity value is given by $\eta_{\max }=B \times \tau_{c}$.

\subsection{Overview of the SPH method}

The SPH key idea on which the method is based is the well-known use of a convolution integral with a Dirac delta function to reproduce a generic function $\mathrm{f}(\underline{\mathrm{x}})$, replaced by a 'bell-shaped' kernel function $W$ [4]. The kernel function is chosen to be non negative, even and with a support domain (usually circular) whose radius is a multiple of a length $h$, named smoothing length. The kernel function is zero outside the support domain and the smoothing length serves as a scaling parameter for its arguments. It also has the property of converging to the Dirac function as the smoothing length approaches to zero. The kernel that has been used for the simulations in the present work is the C4 (quartic) Wendland kernel [7].

\subsection{SPH discretization technique}

The SPH discretization of the mass conservation equation, used in the paper is based essentially on the following equation:

$$
\frac{D \rho_{i}}{D t}=\sum_{j=1}^{n} m_{j} \cdot \underline{v}_{i j} \cdot \nabla_{i} W_{i j}
$$

where $m_{j}$ represents the particle mass; $\underline{v}_{i j}=\underline{v}_{i}-\underline{v}_{j}$ represents the difference between the interacting particles velocity. A widely used SPH discretization of the momentum equation, in the case of Newtonian and non Newtonian viscous fluids, is as follows:

$$
\left.\frac{D \underline{v}_{i}}{D t}=\underline{f}_{i}-\sum_{j=1}^{n} m_{j} \cdot\left(\frac{p_{i}}{\rho_{i}^{2}}+\frac{p_{j}}{\rho_{j}^{2}}\right) \cdot \nabla_{i} W_{i j}+\sum_{j=1}^{n} m_{j} \cdot \frac{16 \eta_{i} \eta_{j}}{\rho_{i} \rho_{j}\left(\eta_{i}+\eta_{j}\right.}\right) \cdot \frac{\underline{v}_{i j} \cdot \underline{r}_{i j}}{\left\|\underline{r}_{i j}\right\|^{2}} \cdot \nabla_{i} W_{i j}
$$

where $\underline{r}_{i j}=\underline{r}_{i}-\underline{r}_{j}$ represents the difference between the interacting particle position; Such an expression has been proposed by Cleary [8] and Monaghan [1] and accounts for the presence of spatial gradients in viscosity too. A possible alternative to eq. (4) is the following SPH approximation for its divergence (for more details, see [4]):

$$
\frac{D \underline{\underline{v}}_{i}}{D t}=\underline{f}_{i}+\sum_{j=1}^{n} m_{j} \cdot\left(\frac{\underline{\underline{\sigma}}}{\rho_{i}^{2}}+\frac{\underline{\sigma}_{j}}{\rho_{j}^{2}}\right) \cdot \nabla_{i} W_{i j}
$$

Another possible formulation for the viscous term was proposed by Morris et al. [9] for the simulation of incompressible viscous flows at low Reynolds numbers. The equation exactly represents the viscous term of the momentum equation up to the 2 nd order. In this paper, a small modification of the viscous term was introduced, yielding the following expression:

$$
\frac{D \underline{v}_{i}}{D t}=\underline{f}_{i}-\sum_{j=1}^{n} m_{j} \cdot\left(\frac{p_{i}}{\rho_{i}^{2}}+\frac{p_{j}}{\rho_{j}^{2}}\right) \cdot \nabla_{i} W_{i j}+\sum_{j=1}^{n} \frac{2 \eta_{i} \eta_{j}}{\left(\eta_{i}+\eta_{j}\right)} \frac{\left(\underline{r}_{i j} \nabla_{i} W_{i j}\right)}{\underline{r}_{i j}^{2} \rho_{i} \rho_{j}} m_{j} v_{i j}
$$

Thus the consequent numerical results were compared and discussed. 
The simulation of an incompressible flow requires the solution of a Poisson equation for the pressure, which often leads to an increase of the computational time. Therefore, it is more practical to approximate the incompressible medium with a weakly compressible one, thus allowing the calculation of the pressure from the density with a stiff equation of state which introduces an artificial compressibility in the fluid. The artificial equation of state used in this paper is based on a selected sound speed, not real but just for numerical purpose:

$$
p_{i}=c^{2}\left(\rho_{i}-\rho_{0}\right) \equiv(\sqrt{g \cdot H} / M)^{2}\left(\rho_{i}-\rho_{0}\right)
$$

where $p_{i}$ is the pressure associated to the $\mathrm{i}$-th particle; $c$ is the artificial speed of sound (the same for all particles); $\mathrm{g}$ is the gravity acceleration; $H$ is the maximum initial dam height; $M$ is the selected value (in input) of Mach number; $\rho_{i}$ is the particle density; $\rho_{0}$ is the reference density of the fluid at zero pressure.

Another important numerical issue is encountered in the computation of shocks waves, especially when low dissipation occurs. Numerical methods, including SPH, develop unphysical oscillations around the fronts of shocks, like a front of a fast flow, unless some special treatment is adopted [4]. A common approach of numerical methods is the one of dissipating the kinetic energy of the shocked region into heat by adding an artificial viscosity term $\Pi_{i j}$ to momentum equation, as proposed by Monaghan and Gingold [10]:

$$
\Pi_{i j}=-v \frac{\underline{v}_{i j} \cdot \underline{r}_{i j}}{\left|\underline{r}_{i j}\right|^{2}} ; v=\frac{\bar{h}_{i j}}{\bar{\rho}_{i j}}\left(\alpha \bar{c}-\beta \frac{\bar{h}_{i j} \underline{v}_{i j} \cdot \underline{r}_{i j}}{\left|\underline{r}_{i j}\right|^{2}}\right)
$$

where $\alpha$ and $\beta$ are non dimensional coefficients, while the over line sign represent arithmetic average between quantities (more details in [4]).

The equations discussed so far are usually referred to as a 'standard' SPH scheme. The momentum equation formulation of the standard scheme is not very diffusive and works well in computing particle positions. Moreover, Ferrari et al. [11] suggest a correction to the continuity equation that both creates a density monotone scheme and leaves substantially unaffected the low diffusivity of the original SPH formulation, by adding a flux term devised from the Rusanov flux [12]:

$$
\frac{D \rho_{i}}{D t}=\sum_{j=1}^{n} m_{j} \cdot \underline{v}_{i j} \cdot \nabla_{i} W_{i j}+\sum_{j=1}^{n} \frac{m_{j}}{\rho_{j}} \cdot c\left(\rho_{i}-\rho_{j}\right) \frac{\nabla_{i} W \cdot \underline{r}_{i j}}{\left|\underline{r}_{i j}\right|}
$$

The SPH approach requires particular care regarding boundary conditions. In this paper, the Ghost Particles (also Image or Wall particles) approach proposed by Morris et al. [9] was selected. In the same article, in order to set no-slip conditions, the following extrapolated 'virtual' boundary particles velocities were given:

$$
\underline{\mathbf{v}}_{\text {bound }}=-\theta \cdot \underline{\mathbf{v}}_{\text {free }} ; \theta=\min \left(\theta_{\max }, 1+d_{w} / d_{f}\right)
$$

where $\theta_{\max }=1.5$ and $d_{w}, d_{f}$ are, respectively, the normal distance of the involved wall particle and the selected free particle from the boundary. Time 
integration has been performed by means of a symplectic Verlet scheme, as in [13]. The time step $\Delta t$ is controlled by a CFL (Courant-Friedrichs, Lewy) condition depending on the artificial speed of sound, the viscous interactions between particles and on the interactions with boundary particles (for detailed discussion see [4]).

\section{Experimental test; consideration about data comparison}

The ability of the selected SPH approaches to correctly reproduce a fast mudflow has been tested by simulating the experiments performed by Laigle and Coussot [5] (L\&C). The experimental setup they used is briefly sketched in the fig. 1. After the opening of the gate, the material stored behind it was released (like a dam break) and the three ultrasonic gauges, sketched in the picture, recorded the mudflow front heights in time. L\&C used water-clay mixtures prepared in laboratory with different concentrations in order to recreate mudflows. HerschelBulkley rheological parameters for the used mixtures were fitted to measures, carried out with a rheometer. Table 1 shows the obtained main parameter values. In particular, along with the experimental conditions of Table 1, represent a realistic natural material (with $\rho_{0}=2200 \mathrm{Kg} / \mathrm{m}^{3}, \tau_{c}=900 \mathrm{~Pa}, K=290 \mathrm{~Pa} \cdot \mathrm{s}^{1 / 3}$ ) in a $120 \mathrm{~m}$ long slope, as detailed in [4].

Data on arrival times for the selected test are affected by experimental errors, ranging from \pm 5 to $\pm 15 \%$ and up to $\pm 20 \%$ [5]. As a consequence, experimental uncertainties could be large enough to hide possible inaccuracies of the selected numerical models, as indeed is discussed in the cited article. Nevertheless, we tried [4] to devise probabilistic considerations in order to perform comparison between numerical results and available data which are affected by experimental errors. Thus, the 'Performance Index' $I_{N, k}$, based on Gaussian distribution function, was introduced by Pasculli et al. [4]. The real experimental value of debris front arrival time at the $k$-th gauge was assumed to be a random variable, affected by only random errors (assumed to be $\pm 25 \%$ ), belonging to a Gaussian probability density function with a mean $\mu_{k}$ equal to the value assessed from experiments and with the statistical dispersion $\sigma_{k}=0.076 \times \mu_{k}$ (for more details see [4]). Such index aims at normalization with respect to the maximum pdf possible value and is introduced for each gauge arrival time and for each simulation.

Table 1: Rheological characteristics and experimental parameters (from [5]).

\begin{tabular}{|l|l|}
\hline density $\left(\rho_{0}\right)$ & $1410\left(\mathrm{~kg} / \mathrm{m}^{3}\right)$ \\
\hline yield stress $\left(\tau_{\mathrm{c}}\right)$ & $19(\mathrm{~Pa})$ \\
\hline power law index $(\mathrm{n})$ & $1 / 3$ \\
\hline liquid consistency $(\mathrm{K})$ & $3.5\left(\mathrm{~Pa} \cdot \mathrm{s}^{\mathrm{n}}\right)$ \\
\hline Flume slope & $21(\%)$ \\
\hline $\begin{array}{l}\text { Initial height of material } \\
\text { (measured at the gate) }\end{array}$ & $14(\mathrm{~cm})$ \\
\hline
\end{tabular}




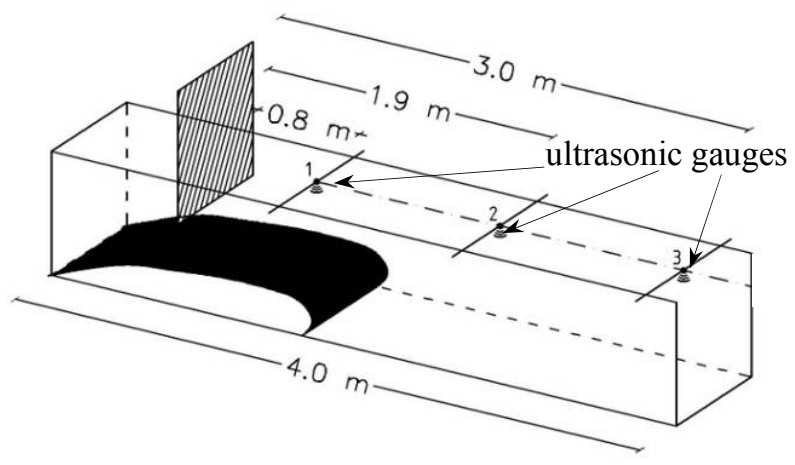

Figure 1: 3D sketch of the selected experimental device (free adapted from [5]).

The following is the proposed expression:

$$
I_{N, k}=\exp \left\{-\frac{\left(X_{n u m, k}-\mu_{k}\right)^{2}}{2 \times\left(0.076 \times \mu_{k}\right)^{2}}\right\}
$$

where $X_{n u m, k}$ was the value of the mudflow front arrival time (also assimilated to a random variable) at the $k$-th gauge, provided by each numerical SPH simulation.

\section{Numerical simulations}

The artificial Mach number was set to a value ranging from 0.03 up to 0.2 . Another important parameter was the initial particles spacing $d p(\mathrm{~mm})$, which played the same role of the grid spacing in finite differences schemes. Since the physics of the flow was dominated by viscosity, the choice of the SPH discretization for the momentum equation played an important role. An important distinctive feature of a SPH model is related to how the selected approach simulates the viscosity interaction among particles. For this reason the performances of three different approaches, that can in principle correctly simulate a viscous non-Newtonian Herschel-Bulkley fluid, were explored. Some main features of the proposed models are briefly summarized below.

Model-0: detailed in [3]; $d p=3 . \mathrm{mm}$; eqn. (5) as momentum equation; Mach number $=0.2$; Monaghan boundary force [14]; no artificial viscosity eqn. (8).

Model-1: momentum equation, eqn. (5); artificial viscosity terms, eqn. (8); Morris wall particles were selected.

Model-2, Monaghan and Cleary approach: some simulations with Rusanov eqn (9); eqn. (4) for linear momentum; Morris wall particles.

Model-3, Morris approach: Rusanov eqn (9); eqn. (6) for linear momentum; Morris wall particles.

In relation to these properties a series of simulations were carried out (for more details see [4]). Table 2 summarizes the main features of the simulations. 
Table 2: Main simulations and numerical results features (free adapted from [4]).

\begin{tabular}{|c|c|c|c|c|c|}
\hline Simul. n. & $\begin{array}{l}\text { Artificial } \\
\text { viscosity }\end{array}$ & Density & $\begin{array}{l}\text { Momentum } \\
\text { equation }\end{array}$ & $\begin{array}{c}\mathrm{dp} \\
(\mathrm{mm})\end{array}$ & Mach \\
\hline 0 & $=$ & $=$ & eqn. 5 & 3. & 0.2 \\
\hline 1 model-1 & $\begin{array}{l}\alpha=0.1 ; \beta=0 . \\
\text { eqn. } 8\end{array}$ & $=$ & eqn. 5 & 2.9 & 0.2 \\
\hline 2 model-1 & $\begin{array}{l}\alpha=0.05 ; \\
\beta=0 . \\
\text { eqn. } 8\end{array}$ & $=$ & eqn. 5 & 2.9 & 0.2 \\
\hline 3 model-2 & $=$ & $=$ & eqn. 4 & 2.9 & 0.2 \\
\hline 4 model-2 & $=$ & $=$ & eqn. 4 & 2.0 & 0.2 \\
\hline 5 model-2 & $=$ & $\begin{array}{c}\text { Rusanov } \\
\text { eqn. } 9\end{array}$ & eqn. 4 & 2.9 & 0.2 \\
\hline 6 model-2 & $=$ & $\begin{array}{c}\text { Rusanov } \\
\text { eqn. } 9\end{array}$ & eqn. 4 & 2.9 & 0.06 \\
\hline 7 model-2 & $=$ & $\begin{array}{c}\text { Rusanov } \\
\text { eqn. } 9\end{array}$ & eqn. 4 & 2.9 & 0.03 \\
\hline 8 model-2 & $=$ & $\begin{array}{c}\text { Rusanov } \\
\text { eqn. } 9\end{array}$ & eqn. 4 & 2.0 & 0.03 \\
\hline 9 model-3 & $=$ & $\begin{array}{c}\text { Rusanov } \\
\text { eqn. } 9\end{array}$ & $\begin{array}{l}\text { Morris } \\
\text { eqn. } 6 ; V_{b}=0 \text {. }\end{array}$ & 2.9 & 0.2 \\
\hline 10 model-3 & $=$ & $\begin{array}{c}\text { Rusanov } \\
\text { eqn. } 9\end{array}$ & $\begin{array}{l}\text { Morris } \\
\text { eqn. 6; } \\
V_{b}=-\theta V_{\text {free }}\end{array}$ & 2.9 & 0.2 \\
\hline
\end{tabular}

\subsection{Results summary}

Fig. 2 shows the direct comparison between numerical arrival times and experimental data, estimated within the range indicated by constant vertical bars and variable error bars. A brief discussion relating to each implemented models follows.

Model-0: this kind of SPH simulation is affected by some numerical diffusion, as the calculated front's velocity is lower than the experimental data. Moreover, from the numerical arrival time at the third gauge, displayed in fig. 2, it is clear that the numerical front experiences unphysical accelerations on the last part of the transitory.

Model-1: differs from Model 0 for the use of artificial viscosity, eqn. (14) and for the use of Morris wall particles instead of Monaghan boundary particles. Simulation 1 differs from Simulation 2 (both using SPH Model 1) for the value of parameter $\alpha$ in eqn. (14) (Table 2). The outcomes of both Simulation 1 and Simulation 2, displayed in fig. 2, show a too diffusive behaviour of the flow. The conclusion is that the Model 1 is not suitable for the case under study.

Model-2: good performances have been observed applying this model. Nevertheless, numerical viscosity related to the spatial resolution (spacing of 
particles) and a bulk viscosity, due to the analytical structure of the model, are introduced by the numerical approach. If a constant dispersion is assumed, only Simulation 8 is roughly within the experimental error.

Model-3: this model, in the light of the theoretical analysis of its features [4], introduces a lower bulk viscosity than Model-2, but, strictly, it is only applicable when the motion is irrotational in the case of a non Newtonian fluid (proof provided in a next paper). Simulations 9 and 10 appear to be more satisfactory than the simulations belonging to Model-2.

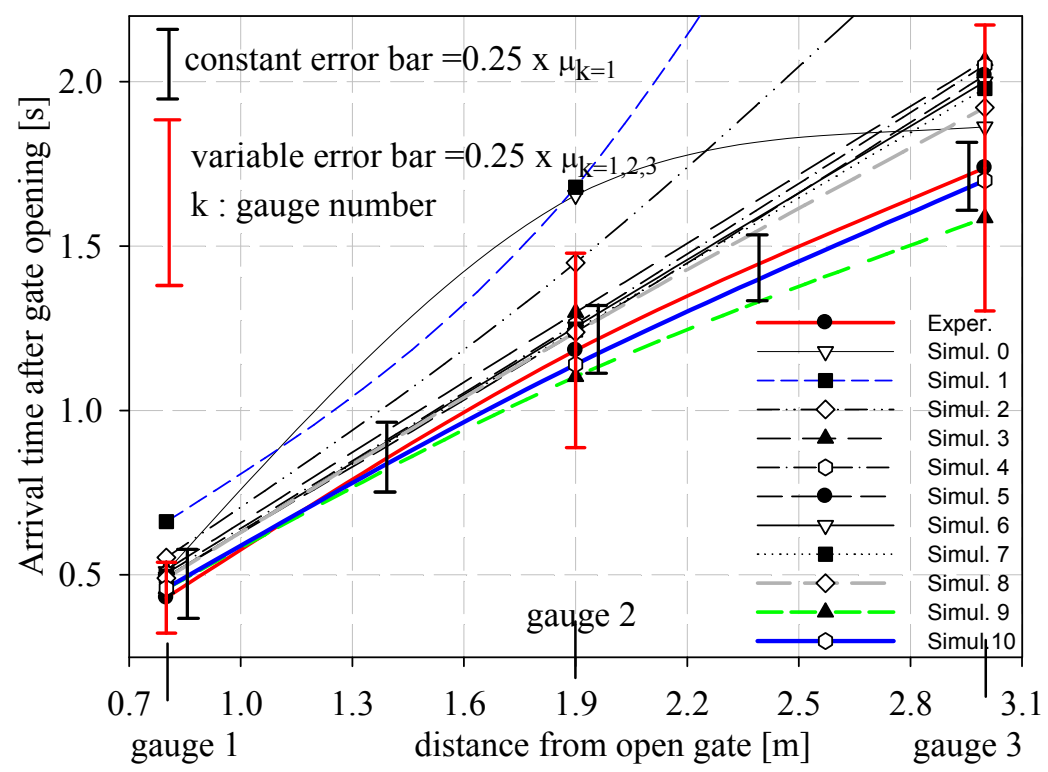

Figure 2: Comparison between numerical arrival times and experimental data (after Pasculli et al. [4]).

Model-3 is less numerically diffusive than Model-2. Simulation 9 differs from Simulation 10 for a different way to calculate the Morris wall particles velocity. In the former case, a null velocity was assigned, while in the latter case an opposite velocity to the one of free particles was assumed $\underline{\mathbf{v}}_{\text {bound }}=-\theta \cdot \underline{\mathbf{v}}_{\text {free }}$. It is worth noting, comparing Simulations 8, 9 and 10, that the increase in the number of particles, not necessarily provides an improvement of performances.

\subsection{Uncertainty of Herschel-Bulkley rheological: sensitive analysis}

Besides the uncertainty on arrival times, significant experimental errors also affect the values of the Herschel-Bulkley rheological parameters characterizing the selected fluid. Typically, uncertainties on the measurements of $\tau_{c}$ and $K$ 
with clay dispersions are rarely less than $20 \%$. Accordingly, in this paper we carried out further analysis to acquire more insights about how much the numerical variability of the parameters requested to apply the selected non Newtonian constitutive rheological equations, describing a viscoplastic material like mudflows, may affect the numerical results of the velocity evolution over time. Hence, we performed different parametric calculations, based on Simul. 10.

The $\tau_{c}$ and $K$ values, related to Herschel-Bulkley rheological law, have been changed from their nominal values, respectively 19 and 3.5. Thus for each of the following three values of the consistency parameter: $K=3.5-0.3 \times 3.5=2.45$, $K=3.5$ and $K=3.5+0.3 \times 3.5=4.55$, three values of the yield stress have been adopted: $\tau_{c}=19-0.3 \times 19=13.3, \tau_{c}=19$ and finally $\tau_{c}=19+0.3 \times 19=24.7$. Fig. 3 shows the numerical results related to three sensitivity analyses varying $\tau_{c}$ around a fixed $K$ value, while fig. 4 shows the graph of the index $I_{N, k}$, calculated for all the performed analyses, while in fig. 5 outcomes of some parameters values combinations are reported. It worth noticing that the index $I_{N, k}$ is indicative of how high is the probability that the numerical result is close to the real value of the arrival time measured from experiments. The rationale of fig. 4 is related to statistical considerations, while figs. 2, 3 are more connected to physical features of the phenomena, as, for example, the acceleration of debris front. The figures show non vanishing effects of this kind of uncertainty which, hence, should be considered within the framework of the simulations of this kind of phenomena.

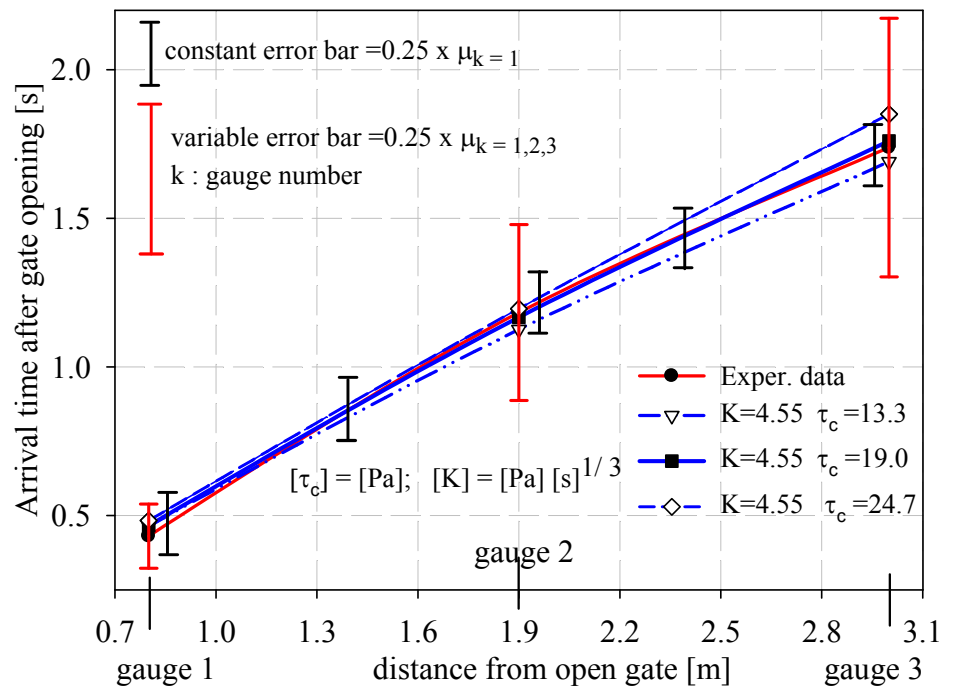

Figure 3: Herschel-Bulkley's parametric analyses: arrival times. 


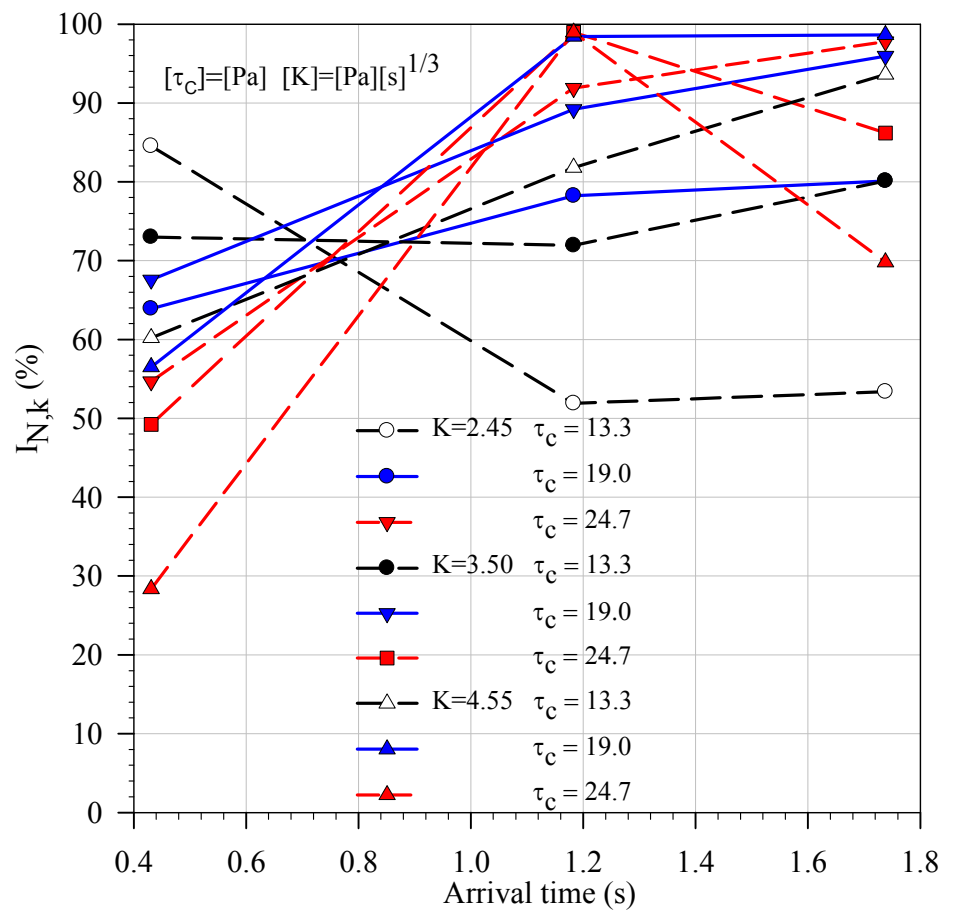

Figure 4: All Herschel-Bulkley's parametric analyses compared to the experimental data through the performance index $I_{N, k}$.

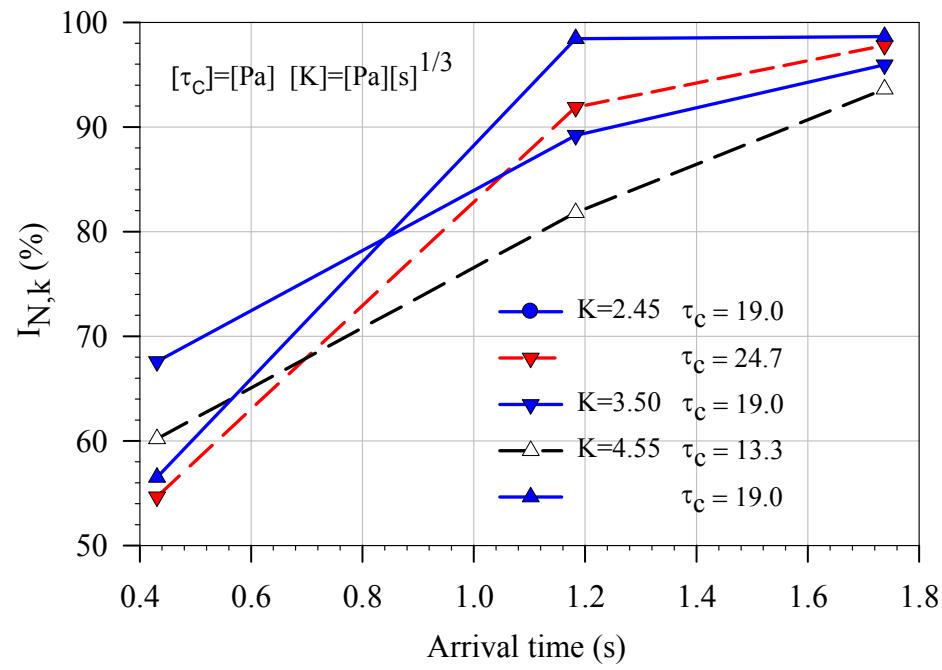

Figure 5: Selected Herschel-Bulkley's parametric analyses compared to the experimental data through the performance index $I_{N, k}$. 


\subsection{Indication of the influence of initial spacing on calculated pressure}

Fig. 6.a shows the numerical pressure distribution after $0.198 \mathrm{~s}$, obtained by Model 2, Simulation 8 (table 2) [4], while fig. 6.b shows the numerical pressure distribution after $0.198 \mathrm{~s}$, based on Model 3 and Simulation 10, table 2, renamed Simulation 11, with the initial spacing $d p$ lowered from $2.9 \mathrm{~mm}$ to $2 \mathrm{~mm}$. By inspection of the figures (within which some iso-pressure lines are sketched), it should be recognize that some boundary effects are present, causing small oscillations in pressure values close to the bottom of the flume. Moreover different pressure distributions emerge, highlighting a sensitiveness about modelling, which will require further investigations.

\section{Conclusion}

In this paper, some parametric analyses were discussed in order to explore how the calculated arrival time at each gauges of the selected experimental test depends on the numerical values uncertainty of the parameters characterizing the Herschel-Bulkely's rheological law. Besides the correct selection of the most suitable SPH approach regarding in particular boundary conditions, a proper choice of the numerical rheological parameters should be pursued. Moreover, through a proposed index, it appears that the performance of the discussed simulations is poor at the starting of the transitory. Also, a demand of a better care on calculation of internal flow pressure distribution or, at least, more investigations about this issue should be advisable.

\begin{tabular}{lllllll}
0 & 200 & 400 & 600 & 800 & 1200 \\
\hline$[\mathrm{Pa}]$ & & & & & \\
\hline
\end{tabular}
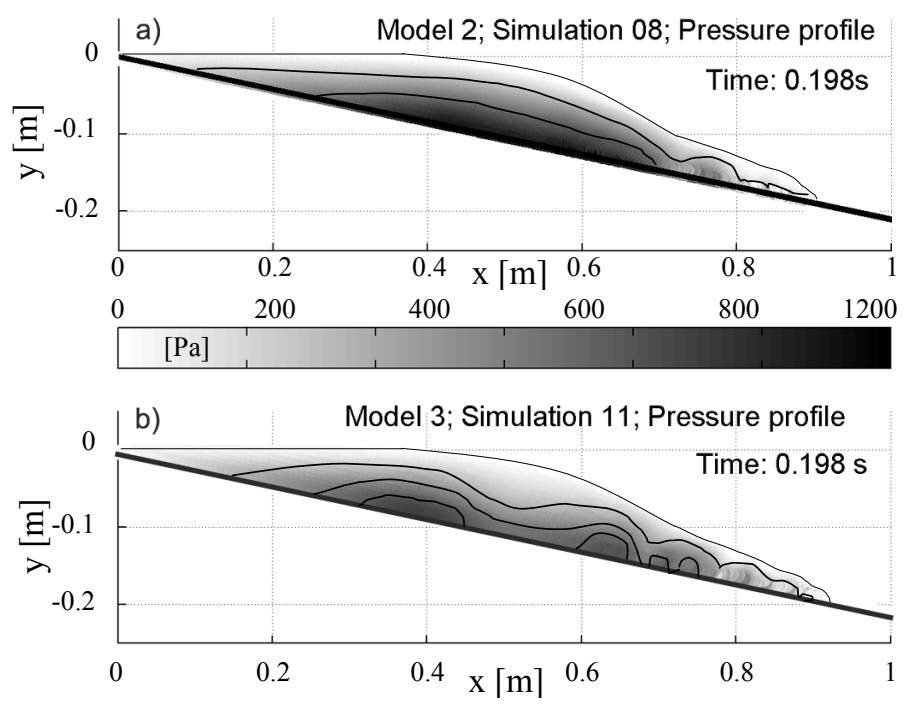

Figure 6: $\quad$ Pressure profiles of particles according to their pressure [Pa]. 


\section{Acknowledgement}

This work was supported by Miur funds, under PRIN 2010-2011 (Dept of Engineering and Geology, Univ. of G. d'Annunzio Chieti-Pescara).

\section{References}

[1] Monaghan, J.J., Smoothed particle hydrodynamics. Reports on Progress in Physics, 68, 1703-1759, 2005.

[2] Minatti, L. \& Pasculli, A., Dam break Smoothed Particle Hydrodynamic modeling based on Riemann solvers. WIT Transactions on Engineering Sciences, 69, 145-156, 2010. ISBN: 978-184564476-5, doi: $10.2495 / A F M 100131$.

[3] Minatti, L., Pasculli, A., SPH numerical approach in modelling 2D muddy debris flow. International Conference on Debris-Flow Hazards Mitigation: Mechanics, Prediction, and Assessment, Proceedings, 467-475, 2011. ISBN: 978-889581446-9, doi: 10.4408/IJEGE.2011-03.B-052.

[4] Pasculli, A., Minatti, L., Sciarra, N., Paris, E., SPH modeling of fast muddy debris flow: Numerical and experimental comparison of certain commonly utilized approaches. Italian Journal of Geosciences, 132 (3), 350-365, 2013.

[5] Laigle, D., \& Coussot, P., Numerical modelling of mudflows. Journal of Hydraulic Engineering, 123(7), 617-623, 1997.

[6] Papanastasiou, T.C., Numerical modelling of mudflows. Journal of Rheology, 31(5), 385, 1987.

[7] Wendland, H., Piecewise polynomial, positive definite and compactly supported radial functions of minimal degree. Advances in Computational Mathematics, 4, 389-396, 1995.

[8] Cleary, P.W., Modelling confined multi-material heat and mass flows using SPH. Appl. Math. Modelling, 22, 981-993, 1998.

[9] Morris, J.P. Fox, P.J. \& ZHU, Y., Modeling low Reynolds number incompressible flows using SPH. Journal of Computational Physics, 136, 214-226, 1997.

[10] Monaghan, J.J. \& Gingold, R.A., Shock simulation by the particle method SPH. Journal of Computational Physics, 52(2), 374-389, 1983.

[11] Ferrari, A., Dumbser, M., Toro, E.F., Armanini, A., A new 3D parallel scheme for free surface flows. Computers \& Fluids, 38, 1203-1217, 2009.

[12] Vila, J.P., On particle weighted methods and smooth particle hydrodynamics. Math Model Methods Appl Sci, 9, 161-209, 1999.

[13] Kajtar, J.B. \& Monaghan, J.J., SPH simulations of swimming linked bodies. Journal of Computational Physics, 227, 8568-8587, 1999.

[14] Monaghan, J.J. \& Kajtar, J.B., SPH particle boundary forces for arbitrary boundaries. Computer Physics Communications, 180, 1811-1820, 2009. 